One limitation of the study was the short follow-up period of 12 months. The authors also noted that they had designed the study before popular procedures such as implantation of tension-free vaginal tape had become widely used.

Original article Corcos J et al. (2005) Multicenter randomized clinical trial comparing surgery and collagen injections for treatment of female stress urinary incontinence. Urology 65: 898-904

\section{RON and MET co-expression is a prognostic indicator in bladder cancer patients}

Protein tyrosine kinases are vital to many cell regulatory processes. The majority of known PROTO-ONCOGENES expressed in solid tumors encode protein tyrosine kinases. Understanding their expression patterns could aid cancer prognosis and treatment. Cheng and co-workers have found a link between decreased survival in bladder cancer patients and expression of the receptor tyrosine kinase RON, the c-met protein (MET), or both.

The group studied the biologic effects of RON overexpression in vitro, and retrospectively analyzed RON and MET expression in 183 bladder cancer patients. RON was found to have a significant effect on cell patterns, increasing proliferation, motility, and antiapoptosis of cells. In addition, histologic grade, larger tumor size, and nonpapillary contour were positively associated with RON and MET expression. RON overexpression was also associated with tumor stage, and increased MET levels with muscle invasion (all $P<0.01)$. RON and MET were overexpressed in $32.8 \%$ and $44.8 \%$ of tumors, respectively; their co-expression led to significantly decreased overall or metastasis-free survival in 35 cases $(19.1 \%)(P=0.005$ and $P=0.01$, respectively). Overexpression of MET alone was also associated with poor patient survival $(P=0.06)$.

The authors conclude that RON-associated signaling might be highly significant in the progression of bladder carcinogenesis. Patients in need of more intensive therapy might be identified by the evaluation of MET and RON expression.

Original article Cheng H-L et al. (2005) Co-expression of RON and MET is a prognostic indicator for patients with transitionalcell carcinoma of the bladder. Br J Cancer 92: 1906-1914

\section{Decreased PTV margin with hypofractionated radiotherapy for prostate cancer}

The standard dose of radiotherapy used to treat prostate cancer is $\leq 70 \mathrm{~Gy}$. Studies suggest that fractions at a higher dose could increase biochemical control rates, especially in patients with high-risk or medium-risk disease. In addition, HYPOFRACTIONATED RADIOTHERAPY could result in more efficient killing of cancer cells. Without high-precision radiotherapy, however, toxicities could be increased significantly.

In their recent study, Cheung et al. investigated whether decreasing the PTV MARGIN could limit the irradiation of noncancerous tissues. Patients were treated in two phases. First, 3D conformal radiotherapy was used to deliver a dose of $2 \mathrm{~Gy} /$ day for 21 days; a $10 \mathrm{~mm}$ PTV margin was used for all patients. Second, an intensity-modulated radiotherapy boost of $3 \mathrm{~Gy} /$ day was administered for 10 days. During this phase, prostate position was measured in each patient using online imaging and implanted FIDUCIAL MARKERS, and PTV margins were adjusted daily (average 3-4 mm).

Of the 33 patients who had completed treatment (7 low-risk, 26 intermediate-risk), none developed $\mathrm{NCI}-\mathrm{CTC}$ GRADE 3 rectal toxicity. Worryingly, three patients developed acute Grade 3 urinary frequency and urgency-a higher number than reported in trials using conventional strategies. The authors conclude, however, that a hypofractionated boost of radiotherapy delivered using high-precision intensitymodulated radiotherapy results in acceptable levels of acute toxicity. Moreover, by using daily online correction, PTV margins can be reduced significantly. Results from other ongoing trials might lead to a greater understanding of the effects on late toxicity and treatment efficacy.

Original article Cheung P et al. (2005) Individualized planning target volumes for intrafraction motion during hypofractionated intensity-modulated radiotherapy boost for prostate cancer. Int J Radiat Oncol Biol Phys 62: 418-425

\section{GLOSSARY}

PROTO-ONCOGENE

A normal cellular gene that has become an oncogene through alteration (e.g. by DNA rearrangement or mutation)

HYPOFRACTIONATED RADIOTHERAPY

Radiotherapy that consists of a higher dose per fraction but a smaller number of fractions than standard radiotherapy; aimed at achieving a shorter irradiation period and a lower total dose

PLANNING TARGET VOLUME (PTV) MARGIN

A margin of error calculated to allow sufficient coverage of the prostate during radiotherapy while limiting the irradiation of normal tissues; it allows for prostate motion, variance in set-up, and the specifics of the treatment

FIDUCIAL MARKERS Small markers that are implanted within the tumor area and detected and visualized electronically; used for position verification

NCI-CTC GRADE 3

Considered a severe and undesirable adverse event in accordance with the National Cancer Institute Common Toxicity Criteria version 2.0 